\title{
Uniform Resolvent Estimates for Magnetic Schrödinger Operators and Smoothing Effects for Related Evolution Equations
}

\author{
by
}

Kiyoshi MochIzUKI

\begin{abstract}
We prove uniform resolvent estimates for the magnetic Schrödinger operator in an exterior domain under smallness conditions on the magnetic fields and the scalar potential. The results are then used to obtain smoothing effects for the corresponding Schrödinger and Klein-Gordon evolution equations.
\end{abstract}

2010 Mathematics Subject Classification: Primary 35Q40; Secondary 81Q10. Keywords: magnetic Schrödinger operator, smoothing.

\section{$\S 1$. Introduction and results}

Let $\Omega$ be an exterior domain in $\mathbb{R}^{n}(n \geq 3)$ with star-shaped complement with respect to the origin 0 and smooth boundary $\partial \Omega$ (the case $\Omega=\mathbb{R}^{n}$ is not excluded). In this paper we consider in $\Omega$ the magnetic Schrödinger equation

$$
-\sum_{j=1}^{n}\left\{\partial_{j}+i b_{j}(x)\right\}^{2} u+c(x) u-\kappa^{2} u=f(x), \quad x \in \Omega,
$$

with Dirichlet boundary condition

$$
u(x, \kappa)=0, \quad x \in \partial \Omega
$$

Here $\partial_{j}=\partial / \partial x_{j}(j=1, \ldots, n), i=\sqrt{-1}, \kappa \in \Pi_{ \pm}=\{\kappa=\sigma+i \epsilon \in \mathbb{C} ; \pm \sigma>0$, $\epsilon>0\}$ and $f \in L^{2}=L^{2}(\Omega) ; b_{j}(x)$ are real-valued $C^{1}$-functions of $x \in \mathbb{R}^{n}$ and $c(x)$ is a real-valued continuous function of $x \in \mathbb{R}^{n} \backslash\{0\} ; b(x)=\left(b_{1}(x), \ldots, b_{n}(x)\right)$ represents a magnetic potential. Thus the magnetic field is defined by its rotation

Communicated by H. Okamoto. Received June 3, 2009. Revised September 15, 2009.

K. Mochizuki: Department of Mathematics, Chuo University, Kasuga, Bunnkyou,

Tokyo 112-8551, Japan;

e-mail: mochizuk@math.chuo-u.ac.jp

(C) 2010 Research Institute for Mathematical Sciences, Kyoto University. All rights reserved. 
$\nabla \times b(x)$. The external potential $c(x)$ may have a singularity like $O\left(|x|^{-2}\right)$ at $x=0$. In this case we assume $c(x)>-\beta / 4|x|^{2}, \beta<(n-2)^{2}$. Throughout this paper, we further require that $\max \{|\nabla \times b(x)|,|c(x)|\}$ decays sufficiently fast as $|x| \rightarrow \infty$.

Notation. Let $a \cdot b$ and $a \times b$ respectively denote the inner product and the exterior product of $a, b \in \mathbb{R}^{n}$. More generally, we put

$$
\nabla \cdot v(x)=\partial_{1} v_{1}(x)+\cdots+\partial_{n} v_{n}(x), \quad \nabla \times v(x)=\left(\partial_{j} v_{k}(x)-\partial_{k} v_{j}(x)\right)_{1 \leq j<k \leq n}
$$

for $\nabla=\left(\partial_{1}, \ldots, \partial_{n}\right)$ and $v(x)=\left(v_{1}(x), \ldots, v_{n}(x)\right)$. We also put $r=|x|, \tilde{x}=x / r$ and $\partial_{r}=\partial / \partial r=\tilde{x} \cdot \nabla$. The inner product and norm of $L^{2}$ are denoted by

$$
(f, g)=\int f(x) \overline{g(x)} d x \text { and } \quad\|f\|=\sqrt{(f, f)} .
$$

Here $\int d x$ indicates integration over the domain $\Omega$. Moreover, for $\rho>0$ we put $\Omega_{\rho}=\{x \in \Omega ;|x|<\rho\}$ and $S_{\rho}=\{x \in \bar{\Omega} ;|x|=\rho\}(\bar{\Omega}=\Omega \cup \partial \Omega)$. We are thus able to consider $\int_{\Omega_{\rho}} d x=\int_{0}^{\rho} \int_{S_{\sigma}} d S d \sigma$.

Now, putting $\nabla_{b}=\nabla+i b(x)$ and $\Delta_{b}=\nabla_{b} \cdot \nabla_{b}$, we define in the Hilbert space $L^{2}$ the operator $L$ as follows:

(3) $\left\{\begin{array}{l}L u=-\Delta_{b} u+c(x) u \text { for } u \in \mathcal{D}(L), \\ \mathcal{D}(L)=\left\{u \in L^{2} \cap H_{\mathrm{loc}}^{2}(\bar{\Omega} \backslash\{0\}) ;-\Delta_{b} u+c u \in L^{2}, \nabla_{b} u \in\left[L^{2}\right]^{n},\left.u\right|_{\partial \Omega}=0\right\} .\end{array}\right.$

Here $H^{j}=H^{j}(\Omega)(j=1,2, \ldots)$ is the usual Sobolev space on $\Omega$ and $H_{\text {loc }}^{2}(\bar{\Omega} \backslash\{0\})$ is the space of $H^{2}$ functions on each compact subset of $\bar{\Omega} \backslash\{0\}$.

Note that the Hardy inequality is easily modified as

$$
\int \frac{(n-2)^{2}}{4 r^{2}}|u|^{2} d x \leq \int\left|\tilde{x} \cdot \nabla_{b} u\right|^{2} d x .
$$

Then, as the Friedrichs extension of a lower semi-bounded symmetric operator $-\Delta_{b}+c$ initially defined on $C_{0}^{\infty}(\Omega \backslash\{0\}), L$ forms a selfadjoint operator in $L^{2}$ with essential spectrum contained in the half line $[0, \infty)$ (see, e.g., Mochizuki [12] and Kalf et al. [7]). Hence, the resolvent $R\left(\kappa^{2}\right)=\left(L-\kappa^{2}\right)^{-1}$ of $L$ can be defined for each $\kappa \in \Pi_{ \pm}$.

The main purpose of this paper is to show the following theorem.

Theorem 1. Let $u$ be the solution of the problem (1), (2).

(i) Assume that

$$
\max \{|\nabla \times b(x)|,|c(x)|\} \leq \mu(r) \quad \text { in } \Omega,
$$

where $\mu=\mu(r)$ is a smooth function of $r>0$ satisfying

$$
\mu>0, \quad \mu^{\prime} \leq 0, \quad \mu \in L^{1}\left(\mathbb{R}_{+}\right) .
$$


Then

$$
\begin{aligned}
\int\left\{\mu \left(\left|\nabla_{b} u\right|^{2}\right.\right. & \left.\left.+|\kappa u|^{2}\right)-\mu^{\prime} \frac{n-1}{2 r}|u|^{2}\right\} d x \\
& \leq 4\|\mu\|_{L^{1}}^{2} \int \mu^{-1}\left(5|f(x)|^{2}+4|\max \{|\nabla \times b|,|c|\} u|^{2}\right) d x
\end{aligned}
$$

for each $\kappa \in \Pi_{ \pm}$and $f$ satisfying $\left(1+\mu(r)^{-1 / 2}\right) f \in L^{2}$. Here $\|\mu\|_{L^{1}}=$ $\int_{0}^{\infty} \mu(\sigma) d \sigma$.

(ii) Assume that

$$
\max \{|\nabla \times b(x)|,|c(x)|\} \leq \epsilon_{0} r^{-2} \quad \text { in } \Omega,
$$

where $0<\epsilon_{0}<1 / 4 \sqrt{2}$. Then

$$
\int \frac{1}{r^{2}}|u|^{2} d x \leq C_{1} \int r^{2}|f|^{2} d x, \quad C_{1}=\frac{32}{(n-2)^{2}-32 \epsilon_{0}^{2}},
$$

for each $\kappa \in \Pi_{ \pm}$and $f$ satisfying $(1+r) f \in L^{2}$.

(iii) Assume that

$$
\max \{|\nabla \times b(x)|,|c(x)|\} \leq \epsilon_{0} \min \left\{\mu(r), r^{-2}\right\} \quad \text { in } \Omega .
$$

Then

$$
\begin{gathered}
\int\left\{\mu\left(\left|\nabla_{b} u\right|^{2}+|\kappa u|^{2}\right)-\mu^{\prime} \frac{n-1}{2 r}|u|^{2}\right\} d x \leq C_{2} \int \max \left\{\mu^{-1}, r^{2}\right\}|f(x)|^{2} d x, \\
C_{2}=4\left(5+4 \epsilon_{0}^{2} C_{1}\right)\|\mu\|_{L^{1}}^{2}
\end{gathered}
$$

for each $\kappa \in \Pi_{ \pm}$and $f$ satisfying $\max \left\{\mu(r)^{-1 / 2}, 1+r\right\} f \in L^{2}$.

(i) shows that the multiplication operator $\sqrt{\mu(r)}$ is locally $L$-smooth near $\kappa=\infty$. The notion of the smooth perturbation introduced by Kato [8] and the local smoothness condition were used in Mochizuki [1] for non-small complex potentials. (ii) shows the $L$-smoothness of $r^{-1}$. Note that (ii) and (iii) generalize the corresponding results of Kato-Yajima [9] (see also Kuroda [10] and Watanabe $[15]$, where the operator in question is the Laplace operator in $\mathbb{R}^{n}(n \geq 3)$. The Fourier transformation method employed there is not applicable in our case. In this paper our arguments are based on the partial integration method widely used to show the principle of limiting absorption. The weight functions introduced in Mochizuki [12 will play an important role. (i) is a result of precise examination of previous results (see, e.g., [12]-[14]). On the other hand, to show (ii) and (iii) it is necessary to introduce yet another identity (see Lemma 3 of $\S 3$ ). The weight function of [12] is especially effective in this argument. 
Remark 1. Assertion (ii) can be generalized to the potential $c(x)=c_{1}(x)+c_{2}(x)$, where $c_{1}(x)$ is a small function satisfying (A2), and $c_{2}(x)$ is a bounded nonnegative function satisfying

$$
c_{2}(x) \leq \mathrm{Cr}^{-2} \quad \text { for some } C>0 .
$$

In this case, to ensure the solvability of an integral equation corresponding to (1), we further require that 0 is not a resonance of $L$.

As a corollary of Theorem 1, we are able to obtain space-time weighted estimates (smoothing effects) for the Schrödinger evolution equation

$$
i \frac{\partial u}{\partial t}-L u=0, \quad u(0)=f \in L^{2},
$$

and the relativistic Schrödinger evolution equation

$$
i \frac{\partial u}{\partial t}-\sqrt{L+m^{2}} u=0, \quad u(0)=f \in L^{2},
$$

with $m \geq 0$. Note that the smoothing effects for (7) give those for the KleinGordon $(m>0)$ or the wave equation $(m=0)$ in the energy space.

Theorem 2. (i) Assume (A2). Then for $f \in L^{2}$ we have

$$
\left|\int_{0}^{ \pm \infty}\left\|r^{-1} e^{-i t L} f\right\|^{2} d t\right| \leq 2 \sqrt{C_{1}}\|f\|^{2} .
$$

(ii) Assume (A3). Then for $f \in L^{2}$ we have

$$
\left|\int_{0}^{ \pm \infty}\left\|\min \left\{\sqrt{\mu(r)}, r^{-1}\right\} e^{-i t \sqrt{L+m^{2}}} f\right\|^{2} d t\right| \leq 4 \sqrt{m^{2} C_{1}+C_{2}}\|f\|^{2} .
$$

(iii) Assume that $b(x) \equiv 0$ and $c(x) \equiv 0$. Then $L$ reduces to the usual Laplacian

$$
L_{0}=-\Delta, \quad \mathcal{D}\left(L_{0}\right)=H^{2} \cap H_{0}^{1} .
$$

In this case, we have

$$
\left|\int_{0}^{ \pm \infty}\left\|\sqrt{\mu(r)} e^{-i t \sqrt{L_{0}}} f\right\|^{2} d t\right| \leq 8 \sqrt{5}\|\mu\|_{L^{1}}\|f\|^{2} .
$$

Similar results have been obtained by many authors in connection with local smoothing properties (see, e.g., Ben Artzi-Klainerman [1], Yajima [16], CuccagnaSchirmer [2, D'Ancona-Fanelli [3, Erdogan-Goldberg-Schlag [4] and GeorgievStefanov-Tarulli [5]). Note that these works are restricted to the initial value problem and the vector potential $b(x)$ itself is required to be small and to decay sufficiently fast. No such requirement is imposed in our case. The decay conditions similar to (A1) on the magnetic field $\nabla \times b(x)$ have been used in Ikebe-Uchiyama [6] to show growth estimates of generalized eigenfunctions corresponding to each positive spectrum. 
As for the Schrödinger equation (6), we can have the following more general results.

Theorem 3. (i) Assume (A2). Then for $h(t)$ satisfying $r^{-1} h(t) \in L^{2}(\mathbb{R} \times \Omega)$ we have

$$
\left|\int_{0}^{ \pm \infty}\left\|r^{-1} \int_{0}^{t} e^{-i(t-\tau) L} h(\tau) d \tau\right\|^{2} d t\right| \leq C_{1}\left|\int_{0}^{ \pm \infty}\|r h(t)\|^{2} d t\right| .
$$

(ii) Assume (A3). Then for $h(t)$ satisfying $\max \left\{\sqrt{\mu(r)}^{-1}, r\right\} h(t) \in L^{2}(\mathbb{R} \times \Omega)$ we have

$$
\begin{aligned}
& \left|\int_{0}^{ \pm \infty}\left\|\min \left\{\sqrt{\mu(r)}, r^{-1}\right\} \int_{0}^{t} \nabla_{b} e^{-i(t-\tau) L} h(\tau) d \tau\right\|^{2} d t\right| \\
& \leq \max \left\{C_{1}, C_{2}\right\}\left|\int_{0}^{ \pm \infty}\left\|\max \left\{\sqrt{\mu(r)}^{-1}, r\right\} h(t)\right\|^{2} d t\right| .
\end{aligned}
$$

The rest of the paper will be organized as follows. In the next section we prepare two identities related to the solution of (1), (2). They are used in $\S 3$ to prove Theorem 1. Finally, Theorems 2 and 3 are proved in $\S 4$ based on Theorem 1 .

\section{$\S 2$. Functional identities for solutions}

In this section we prepare two functional identities related to the solution of (1), (2).

We first multiply both sides of (1) by $-\overline{i \kappa u}$ to obtain

$$
\nabla \cdot\left\{\left(\nabla_{b} u\right) \overline{i \kappa u}\right\}-\overline{i \kappa}\left\{\left|\nabla_{b} u\right|^{2}+c(x)|u|^{2}-\kappa^{2}|u|^{2}\right\}=-f \overline{i \kappa u} .
$$

Integrating the real part of this equation over $\Omega_{\rho}(\rho>0)$, by using the boundary condition (2) we obtain

$$
\begin{aligned}
\operatorname{Re} \int_{\Omega_{\rho}} \nabla \cdot\left\{\left(\nabla_{b} u\right) \overline{i \kappa u}\right\} d x & =\operatorname{Re} \int_{S_{\rho}}\left(\tilde{x} \cdot \nabla_{b} u\right) \overline{i \kappa u} d S \\
& =\frac{1}{2} \int_{S_{\rho}}\left\{-\left|\nabla_{b} u-i \kappa \tilde{x} u\right|^{2}+\left|\nabla_{b} u\right|^{2}+|\kappa u|^{2}\right\} d S,
\end{aligned}
$$

and it follows that

$$
\begin{aligned}
\frac{1}{2} \int_{S_{\rho}}\left\{-\mid \nabla_{b} u\right. & \left.-\left.i \kappa u\right|^{2}+\left|\nabla_{b} u\right|^{2}+|\kappa u|^{2}\right\} d S \\
& +\operatorname{Im} \kappa \int_{\Omega_{\rho}}\left(\left|\nabla_{b} u\right|^{2}+c|u|^{2}+|\kappa u|^{2}\right) d x=-\operatorname{Re} \int_{\Omega_{\rho}} f \overline{i \kappa u} d x .
\end{aligned}
$$


The following proposition is a direct consequence of this identity multiplied by $\mu(\rho)$ and integrated over $(0, \infty)$.

Proposition 1. Let $u \in \mathcal{D}(L)$ satisfy (1), (2) with $\kappa \in \Pi_{ \pm}$and $f \in L^{2}$. Then for $\mu$ satisfying (5) we have

$$
\begin{aligned}
\frac{1}{2} \int \mu\left\{-\left|\nabla_{b} u-i \kappa \tilde{x} u\right|^{2}+\left|\nabla_{b} u\right|^{2}+|\kappa u|^{2}\right\} & d x \\
+\operatorname{Im} \kappa \int_{0}^{\infty} \mu(\rho) d \rho \int_{\Omega_{\rho}}\left(\left|\nabla_{b} u\right|^{2}+c|u|^{2}+|\kappa u|^{2}\right) d x & \\
& =-\operatorname{Re} \int_{0}^{\infty} \mu(\rho) d \rho \int_{\Omega_{\rho}} f \overline{i \kappa u} d x .
\end{aligned}
$$

Next, we put $v=e^{-i \kappa r} r^{(n-1) / 2} u, g=e^{-i \kappa r} r^{(n-1) / 2} f$ and rewrite (1) as follows:

(9) $\quad-\nabla_{b} \cdot \nabla_{b} v+\left(-2 i \kappa+\frac{n-1}{r}\right) \tilde{x} \cdot \nabla_{b} v+\left(\frac{(n-1)(n-3)}{4 r^{2}}+c\right) v=g$.

Let $\varphi=\varphi(r)$ be a positive increasing function of $r>0$ such that

$$
\varphi(r)=O(r) \text { and } \quad \frac{\varphi^{\prime}(r)}{\varphi(r)} \leq \frac{1}{r},
$$

and let $\phi=\phi(r)=e^{-2 \operatorname{Im} \kappa r} r^{-n+1} \varphi(r)$. We multiply both sides of (9) by $\phi \overline{\tilde{x} \cdot \nabla_{b} v}$ to obtain

$$
\begin{aligned}
-\operatorname{Re} \nabla \cdot\{ & \left.\left(\phi \nabla_{b} v\right) \overline{\tilde{x} \cdot \nabla_{b} v}\right\}+\phi^{\prime}\left|\tilde{x} \cdot \nabla_{b} v\right|^{2}+\frac{\phi}{r}\left(\left|\nabla_{b} v\right|^{2}-\left|\tilde{x} \cdot \nabla_{b} v\right|^{2}\right) \\
& +\frac{1}{2} \nabla \cdot\left(\phi \tilde{x}\left|\nabla_{b} v\right|^{2}\right)-\left(\frac{\phi^{\prime}}{2}+\phi \frac{n-1}{2 r}\right)\left|\nabla_{b} v\right|^{2} \\
& -\operatorname{Re} \phi\left\{\left(\tilde{x} \times \nabla_{b} v\right) \cdot \overline{(\nabla \times i b) v}\right\}+\phi\left(2 \operatorname{Im} \kappa+\frac{n-1}{r}\right)\left|\tilde{x} \cdot \nabla_{b} v\right|^{2} \\
& +\operatorname{Re} \phi\left(\frac{(n-1)(n-3)}{4 r^{2}}+c\right) v \overline{\tilde{x} \cdot \nabla_{b} v}=\operatorname{Re}\left\{\phi g \overline{\tilde{x} \cdot \nabla_{b} v}\right\} .
\end{aligned}
$$

We integrate this over $\Omega_{\rho}$. Then noting

$$
\phi^{\prime}(r)=\phi(r)\left(-2 \operatorname{Im} \kappa-\frac{n-1}{r}+\frac{\varphi^{\prime}}{\varphi}\right)
$$

and

$$
\begin{aligned}
\operatorname{Re} \phi \frac{(n-1)(n-3)}{4 r^{2}} v \overline{\tilde{x} \cdot \nabla_{b} v} & =\frac{1}{2} \nabla \cdot\left\{\phi \tilde{x} \frac{(n-1)(n-3)}{4 r^{2}}|v|^{2}\right\} \\
+\phi\left(\operatorname{Im} \kappa-\frac{\varphi^{\prime}}{2 \varphi}\right) & \frac{(n-1)(n-3)}{4 r^{2}}|v|^{2}+\phi \frac{(n-1)(n-3)}{4 r^{3}}|v|^{2},
\end{aligned}
$$

we obtain the following proposition. 
Proposition 2. Let $v$ satisfy (9) with boundary condition $\left.v\right|_{\partial \Omega}=0$. Then

$$
\begin{aligned}
& \int_{S_{\rho}} \phi\left\{-\left|\tilde{x} \cdot \nabla_{b} v\right|^{2}+\frac{1}{2}\left|\nabla_{b} v\right|^{2}+\frac{1}{2} \frac{(n-1)(n-3)}{4 r^{2}}|v|^{2}\right\} d S \\
& \quad+\int_{\partial \Omega \cap\{|x|<\rho\}} \phi\left\{-\left(\nu \cdot \nabla_{b} v\right)\left(\overline{\tilde{x} \cdot \nabla_{b} v}\right)+\frac{1}{2}(\nu \cdot \tilde{x})\left|\nabla_{b} v\right|^{2}\right\} d S \\
& \quad+\int_{\Omega_{\rho}} \phi\left\{\left(\frac{1}{r}-\frac{\varphi^{\prime}}{\varphi}\right)\left(\left|\nabla_{b} v\right|^{2}-\left|\tilde{x} \cdot \nabla_{b} v\right|^{2}+\frac{(n-1)(n-3)}{4 r^{2}}|v|^{2}\right)\right. \\
& \quad+\left(\operatorname{Im} \kappa+\frac{\varphi^{\prime}}{2 \varphi}\right)\left(\left|\nabla_{b} v\right|^{2}+\frac{(n-1)(n-3)}{4 r^{2}}|v|^{2}\right) \\
& \left.\quad+\operatorname{Re}\left[-\left(\tilde{x} \times \nabla_{b} v\right) \cdot \overline{(\nabla \times i b) v}+c v \overline{\tilde{x} \cdot \nabla_{b} v}\right]\right\} d x=\operatorname{Re} \int_{\Omega_{\rho}} \phi g \overline{\tilde{x} \cdot \nabla_{b} v} d x .
\end{aligned}
$$

where $\nu=\nu(x)$ is the outer unit normal to the boundary $\partial \Omega$.

\section{§3. Proof of Theorem 1}

We shall show Theorem 1 by a series of lemmas.

Lemma 1. Assume $c(x) \geq-(n-2)^{2} / 4 r^{2}$. Then for $\mu$ satisfying (5) we have

$$
\begin{aligned}
& \frac{1}{2} \int\left\{\mu \operatorname{Im} \kappa \frac{1}{r}|u|^{2}-\mu^{\prime} \frac{n-1}{2 r}|u|^{2}+\mu\left(\left|\nabla_{b} u\right|^{2}+|\kappa u|^{2}\right)\right\} d x \\
& \leq \frac{1}{2} \int \mu\left(|\theta|^{2}+\frac{(n-1)(n-3)}{4 r^{2}}|u|^{2}\right) d x+\|\mu\|_{L^{1}} \int|f(x)||i \kappa u| d x
\end{aligned}
$$

where

$$
\theta=\nabla_{b} u+\tilde{x}\left(\frac{n-1}{2 r}-i \kappa\right) u
$$

Proof. Note that

$$
\begin{aligned}
\mu\left|\nabla_{b} u-i \kappa \tilde{x} u\right|^{2}= & -\nabla \cdot\left\{\tilde{x} \mu \frac{n-1}{2 r}|u|^{2}\right\}+\mu\left(|\theta|^{2}+\frac{(n-1)(n-3)}{4 r^{2}}|u|^{2}\right) \\
& +\mu^{\prime} \frac{n-1}{2 r}|u|^{2}-\mu \operatorname{Im} \kappa \frac{n-1}{r}|u|^{2}
\end{aligned}
$$

and

$$
\begin{aligned}
\left|\nabla_{b} u\right|^{2}+c|u|^{2} & \geq\left|\tilde{x} \cdot \nabla_{b} u\right|^{2}-\frac{(n-2)^{2}}{4 r^{2}}|u|^{2} \\
& =\left|\tilde{x} \cdot \nabla_{b} u+\frac{n-2}{2 r} u\right|^{2}-\nabla \cdot\left(\tilde{x} \frac{n-2}{2 r}|u|^{2}\right) .
\end{aligned}
$$


in Proposition 1. Then since

$$
\liminf _{\rho \rightarrow \infty} \int_{S_{\rho}} \mu \frac{n-1}{2 r}|u|^{2} d S=0
$$

we have

$$
\begin{aligned}
- & \frac{1}{2} \int \mu\left|\nabla_{b} u-i \kappa \tilde{x} u\right|^{2} d x+\operatorname{Im} \kappa \int_{0}^{\infty} \mu(\rho) d \rho \int_{\Omega_{\rho}}\left(\left|\nabla_{b} u\right|^{2}+c|u|^{2}\right) d x \\
\geq & -\frac{1}{2} \int\left\{\mu\left(|\theta|^{2}+\frac{(n-1)(n-3)}{4 r^{2}}|u|^{2}\right)+\mu^{\prime} \frac{n-1}{2 r}|u|^{2}-\mu \operatorname{Im} \kappa \frac{n-1}{r}|u|^{2}\right\} d x \\
& +\operatorname{Im} \kappa \int_{0}^{\infty} \mu(\rho) d \rho \int_{\Omega_{\rho}}-\nabla \cdot\left(\tilde{x} \frac{n-2}{2 r}|u|^{2}\right) d x \\
= & -\frac{1}{2} \int\left\{\mu\left(|\theta|^{2}+\frac{(n-1)(n-3)}{4 r^{2}}|u|^{2}\right)+\mu^{\prime} \frac{n-1}{2 r}|u|^{2}-\mu \operatorname{Im} \kappa \frac{1}{r}|u|^{2}\right\} d x
\end{aligned}
$$

and the desired inequality.

Lemma 2. For $\varphi(r)$ satisfying (10) we have

$$
\begin{aligned}
\frac{1}{4} \int \varphi\left(\operatorname{Im} \kappa+\frac{\varphi^{\prime}}{2 \varphi}\right)\left(|\theta|^{2}+\frac{(n-1)(n-3)}{4 r^{2}}|u|^{2}\right) d x \\
\leq \int \frac{\varphi^{2}}{\varphi^{\prime}}\left(|f|^{2}+|\max \{|\nabla \times b|,|c|\} u|^{2}\right) d x
\end{aligned}
$$

Proof. In the identity of Proposition 2, the Dirichlet condition (2) implies that $\tau \cdot \nabla v=0$ for any tangential vector $\tau$ to the boundary. On the other hand, since the starshapedness of the boundary implies $\nu \cdot \tilde{x} \leq 0$, it follows that

$\int_{\partial \Omega} \phi\left\{-\left(\nu \cdot \nabla_{b} v\right)\left(\tilde{x} \cdot \overline{\nabla_{b} v}\right)+\frac{1}{2}(\nu \cdot \tilde{x})\left|\nabla_{b} v\right|^{2}\right\} d S=-\frac{1}{2} \int_{\partial \Omega} \phi(\nu \cdot \tilde{x})\left|\nu \cdot \nabla_{b} v\right|^{2} d S \geq 0$.

Moreover, since $1 / r \geq \varphi^{\prime} / \varphi,\left|\nabla_{b} v\right| \geq\left|\tilde{x} \cdot \nabla_{b} v\right|, n \geq 3$ and

$$
\left|-\left(\tilde{x} \times \nabla_{b} v\right) \cdot \overline{(\nabla \times i b) v}+c v \overline{\tilde{x} \cdot \nabla_{b} v}\right| \leq|\max \{|\nabla \times b|,|c|\} v|\left|\nabla_{b} v\right|,
$$

it follows that

$$
\begin{aligned}
\int_{\Omega_{\rho}} \phi & \left(\operatorname{Im} \kappa+\frac{\varphi^{\prime}}{2 \varphi}\right)\left(\left|\nabla_{b} v\right|^{2}+\frac{(n-1)(n-3)}{4 r^{2}}|v|^{2}\right) d x \\
\leq & \int_{\Omega_{\rho}} \phi(|g|+|\max \{|\nabla \times b|,|c|\} v|)\left|\nabla_{b} v\right| d x+\frac{1}{2} \int_{S_{\rho}} \phi\left|\tilde{x} \cdot \nabla_{b} v\right|^{2} d S .
\end{aligned}
$$

If we let $\rho \rightarrow \infty$, then $\varphi(r)=O(r)$ leads to

$$
\liminf _{\rho \rightarrow \infty} \int_{S_{\rho}} \phi\left|\tilde{x} \cdot \nabla_{b} v\right|^{2} d S=\liminf _{\rho \rightarrow \infty} \int_{S_{\rho}} \varphi|\tilde{x} \cdot \theta|^{2} d S=0
$$

and we obtain the inequality of the lemma. 
Proof of Theorem 1(i). We combine Lemmas 1 and 2 with $\varphi(r)=\int_{0}^{r} \mu(\sigma) d \sigma$. It is obvious that this $\varphi$ satisfies (10). Then since $\varphi(r) \leq\|\mu\|_{L^{1}}$, it follows that

$$
\begin{aligned}
& \frac{1}{2} \int\left\{-\mu^{\prime} \frac{n-1}{2 r}|u|^{2}+\mu\left(\left|\nabla_{b} u\right|^{2}+|\kappa u|^{2}\right)\right\} d x \\
& \quad \leq 4\|\mu\|_{L^{1}}^{2} \int \mu^{-1}\left(|f|^{2}+|\max \{|\nabla \times b|,|c|\} u|^{2}\right) d x+\|\mu\|_{L^{1}} \int|f||i \kappa u| d x .
\end{aligned}
$$

Thus, noting

$$
\|\mu\|_{L^{1}} \int|f||i \kappa u| d x \leq\|\mu\|_{L^{1}}^{2} \int \mu^{-1}|f|^{2} d x+\frac{1}{4} \int \mu|\kappa u|^{2} d x
$$

we deduce the desired inequality.

To complete the proof of (ii) and (iii), we need one more lemma.

Lemma 3. We have

$$
\int(2 \operatorname{Im} \kappa r+1) \frac{1}{4 r^{2}}|u|^{2} d x \leq \int(2 \operatorname{Im} \kappa r+1)|\tilde{x} \cdot \theta|^{2} d x .
$$

Proof. We put $\psi=e^{-\operatorname{Im} \kappa r}(2 \operatorname{Im} \kappa r+1)^{1 / 2}$. Then we have

$$
(2 \operatorname{Im} \kappa r+1)|\tilde{x} \cdot \theta|^{2}=r^{-n+1} \psi^{2}\left|\tilde{x} \cdot \nabla_{b} v\right|^{2} .
$$

Note that

$$
\begin{aligned}
\psi^{2}\left|\tilde{x} \cdot \nabla_{b} v\right|^{2}= & \left|\tilde{x} \cdot \nabla_{b}(\psi v)-\psi^{\prime} v\right|^{2}=\left|\left\{\tilde{x} \cdot \nabla_{b}(\psi v)-\frac{\psi^{\prime}}{2} v-\xi v\right\}-\frac{\psi^{\prime}}{2} v+\xi v\right|^{2} \\
= & \left|\tilde{x} \cdot \nabla_{b}(\psi v)-\frac{\psi^{\prime}}{2} v-\xi v\right|^{2}-\partial_{r}\left\{\left(\frac{\psi^{\prime}}{2 \psi}-\frac{\xi}{\psi}\right)|\psi v|^{2}\right\} \\
& +\left(\frac{\psi^{\prime \prime} \psi-\psi^{\prime 2}}{2}-\xi^{\prime} \psi+\xi \psi^{\prime}\right)|v|^{2}+2\left(\frac{\psi^{\prime 2}}{4}-\xi^{2}\right)|v|^{2}+\left|\frac{\psi^{\prime}}{2} v-\xi v\right|^{2} \\
= & \left|\tilde{x} \cdot \nabla_{b}(\psi v)-\frac{\psi^{\prime}}{2} v-\xi v\right|^{2}-\partial_{r}\left\{\left(\frac{\psi^{\prime}}{2 \psi}-\frac{\xi}{\psi}\right)|\psi v|^{2}\right\} \\
& +\frac{1}{2}\left(\frac{1}{2} \psi^{\prime 2}+\psi^{\prime \prime} \psi-2 \xi^{\prime} \psi-2 \xi^{2}\right)|v|^{2},
\end{aligned}
$$

where $\xi=\xi(r)$ is another weight function given later. By definition we have

$$
\psi^{\prime}=\psi \frac{-2(\operatorname{Im} \kappa)^{2} r}{2 \operatorname{Im} \kappa r+1}<0, \quad \psi^{\prime \prime} \psi=\psi^{\prime 2}+\frac{1}{r(2 \operatorname{Im} \kappa r+1)} \psi^{\prime} \psi .
$$

Then since

$$
\frac{1}{2} \psi^{\prime 2}+\psi^{\prime \prime} \psi=\frac{3}{2} \psi^{\prime 2}+\left\{\frac{1}{r(2 \operatorname{Im} \kappa r+1)}-\frac{1}{r}\right\} \psi^{\prime} \psi+\frac{1}{r} \psi^{\prime} \psi \geq \frac{1}{r} \psi^{\prime} \psi,
$$


we can now choose $\xi=\psi / 2 r$ to obtain

$$
\frac{1}{2} \psi^{\prime 2}+\psi^{\prime \prime} \psi-2 \xi^{\prime} \psi-2 \xi^{2} \geq \frac{1}{r} \psi^{\prime} \psi-2 \xi^{\prime} \psi-2 \xi^{2}=\frac{1}{2 r^{2}} \psi^{2},
$$

and it follows that

$$
r^{-n+1} \psi^{2}\left|\tilde{x} \cdot \nabla_{b} v\right|^{2} \geq \nabla \cdot\left\{\tilde{x}\left(-\frac{\psi^{\prime}}{2 \psi}+\frac{1}{2 r}\right) r^{-n+1}|\psi v|^{2}\right\}+\frac{\psi^{2}}{4 r^{2}} r^{-n+1}|v|^{2} .
$$

Integrate both sides over $\Omega_{\rho}$. Then since

$$
\int_{S_{\rho}}\left(-\frac{\psi^{\prime}}{2 \psi}+\frac{1}{2 r}\right) r^{-n+1}|\psi v|^{2} d S=O(\rho) \int_{S_{\rho}}|u|^{2} d S
$$

letting $\rho \rightarrow \infty$, we deduce the inequality of the lemma.

Proof of Theorem 1(ii). We choose $\varphi=r$ in Lemma 2. Then noting (A2), we have

$$
\int(2 \operatorname{Im} \kappa r+1)|\theta|^{2} d x \leq 8 \int\left(r^{2}|f|^{2}+\epsilon_{0}^{2} r^{-2}|u|^{2}\right) d x .
$$

Combining this and Lemma 3, we obtain the inequality of (ii).

Proof of Theorem 1(iii). (A3) and the inequality of (ii) imply that

$$
\int \mu^{-1}|\max \{|\nabla \times b|,|c|\} u|^{2} d x \leq \epsilon_{0}^{2} \int \mu^{-1}\left|\min \left\{\mu, r^{-2}\right\} u\right|^{2} d x \leq \epsilon_{0}^{2} C_{1} \int r^{2}|f|^{2} d x .
$$

Substituting this in the inequality of (i), we obtain the desired inequality.

\section{§4. Proof of Theorems 2 and 3}

First we shall summarize abstract results which allow us to employ the resolvent estimate for a selfadjoint operator to show a space-time weighted estimate for the associated evolution equation.

Let $\Lambda$ be a selfadjoint operator in the Hilbert space $\mathcal{H}$, and for $z \in \mathbb{C} \backslash \mathbb{R}$ let $\mathcal{R}(z)$ be the resolvent of $\Lambda$. Suppose that $A$ is a densely defined, closed operator from $\mathcal{H}$ to another Hilbert space $\mathcal{H}_{1}$.

Proposition 3. Assume that there exists $C>0$ such that

$$
\sup _{z \notin \mathbb{R}}\left\|A \mathcal{R}(z) A^{*} f\right\|_{\mathcal{H}_{1}}<\sqrt{C}\|f\|_{\mathcal{H}_{1}}
$$


for $f \in \mathcal{D}\left(A^{*}\right)$. Then

$$
\begin{aligned}
\left|\int_{0}^{ \pm \infty}\left\|\int_{0}^{t} A e^{-i(t-\tau) \Lambda} A^{*} h(\tau) d \tau\right\|_{\mathcal{H}_{1}}^{2} d t\right| & \leq C\left|\int_{0}^{ \pm \infty}\|h(t)\|_{\mathcal{H}_{1}}^{2} d t\right|, \\
\sup _{t \in \mathbb{R}_{ \pm}}\left\|\int_{0}^{t} e^{i \tau \Lambda} A^{*} h(\tau) d \tau\right\|_{\mathcal{H}^{2}}^{2} & \leq 2 \sqrt{C}\left|\int_{0}^{ \pm \infty}\|h(t)\|_{\mathcal{H}_{1}}^{2} d t\right|
\end{aligned}
$$

for each $h \in L^{2}\left(\mathbb{R} ; \mathcal{D}\left(A^{*}\right)\right)$, and

$$
\left|\int_{0}^{ \pm \infty}\left\|A e^{-i t \Lambda} f\right\|_{\mathcal{H}_{1}}^{2} d t\right| \leq 2 \sqrt{C}\|f\|_{\mathcal{H}}^{2}
$$

for each $f \in \mathcal{H}$.

Proof. To show (12) and (13), by the standard approximation procedure, we can assume $h \in C_{0}^{\infty}\left(\mathbb{R} ; \mathcal{D}\left(A^{*}\right)\right)$.

We put $v(t)=\int_{0}^{t} e^{-i(t-\tau) \Lambda} A^{*} h(\tau) d \tau$, and consider its Laplace transform

$$
\tilde{v}(z)= \pm \int_{0}^{ \pm \infty} e^{i z t} v(t) d t, \quad \pm \operatorname{Im} z>0 .
$$

Then since $\tilde{v}(z)=-i \mathcal{R}(z) A^{*} \tilde{h}(z)$, it follows from the Plancherel theorem and the assumption (11) that

$$
\begin{aligned}
&\left|\int_{0}^{ \pm \infty} e^{\mp 2 \epsilon t}(A v(t), g(t))_{\mathcal{H}_{1}} d t\right|=\left|(2 \pi)^{-1} \int_{-\infty}^{\infty}(A \tilde{v}(\lambda \pm i \epsilon), \tilde{g}(\lambda \pm i \epsilon))_{\mathcal{H}_{1}} d \lambda\right| \\
& \leq(2 \pi)^{-1} \int_{-\infty}^{\infty}\left\|A \mathcal{R}(\lambda \pm i \epsilon) A^{*} \tilde{h}(\lambda \pm i \epsilon)\right\|_{\mathcal{H}_{1}}\|\tilde{g}(\lambda \pm i \epsilon)\|_{\mathcal{H}_{1}} d \lambda \\
& \leq\left|C \int_{0}^{ \pm \infty} e^{\mp 2 \epsilon t}\|h(t)\|_{\mathcal{H}_{1}}^{2} d t \int_{0}^{ \pm \infty} e^{\mp 2 \epsilon t}\|g(t)\|_{\mathcal{H}_{1}}^{2} d t\right|^{1 / 2}
\end{aligned}
$$

for any $g \in C_{0}^{\infty}\left(\mathbb{R} ; \mathcal{D}\left(A^{*}\right)\right)$. Letting $\epsilon \downarrow 0$, we obtain inequality (12).

Next, note that the Fubini theorem implies

$$
\begin{aligned}
\left\|\int_{0}^{t} e^{i \tau \Lambda} A^{*} h(\tau) d \tau\right\|_{\mathcal{H}_{1}}^{2}= & \int_{0}^{t}\left(\int_{0}^{s} A e^{-i(s-\tau) \Lambda} A^{*} h(\tau) d \tau, h(s)\right)_{\mathcal{H}_{1}} d s \\
& +\int_{0}^{t}\left(h(\tau), \int_{0}^{\tau} A e^{-i(\tau-s) \Lambda} A^{*} h(s) d s\right)_{\mathcal{H}_{1}} d \tau .
\end{aligned}
$$

This and (12) show that (13) holds.

(14) is the dual assertion of (13).

Proof of Theorem 2(i) and Theorem 3(i). Set $\Lambda=L, \mathcal{H}=\mathcal{H}_{1}=L^{2}$ and $A=r^{-1}$ (multiplication operator). Then $A^{*}=A$ and $\mathcal{R}(z)=R(z)$, and if we let $z=\kappa^{2}$, 
then it follows from Theorem 1(ii) that

$$
\left\|A R(z) A^{*} f\right\|=\left\|r^{-1} R(z) A^{*} f\right\| \leq \sqrt{C_{1}}\left\|r A^{*} f\right\|=\sqrt{C_{1}}\|f\| .
$$

Thus, the estimates (12) and (14) can be written as

$$
\begin{gathered}
\left|\int_{0}^{ \pm \infty}\left\|r^{-1} \int_{0}^{t} e^{-i(t-\tau) L} h(\tau) d \tau\right\|^{2} d t\right| \leq C_{1}\left|\int_{0}^{ \pm \infty}\|r h(t)\|^{2} d t\right|, \\
\left|\int_{0}^{ \pm \infty}\left\|r^{-1} e^{-i t L} f\right\|^{2} d t\right| \leq 2 \sqrt{C_{1}}\|f\|^{2},
\end{gathered}
$$

as desired.

Proof of Theorem 3(ii). Put $A=\min \left\{\sqrt{\mu(r)}, r^{-1}\right\}$. Then by Theorem 1(iii) we have

$$
\left\|A \nabla_{b} R(\lambda \pm i \epsilon) A^{*} \tilde{h}(\lambda \pm i \epsilon)\right\| \leq \sqrt{C_{2}}\|\tilde{h}(\lambda \pm i \epsilon)\|
$$

Thus, we can use the argument proving (12) to obtain the desired conclusion.

To show Theorem 2(ii) we consider the Klein-Gordon equation

$$
i \partial_{t} u=\Lambda u, \quad u(t)=\left\{w(t), \partial_{t} w(t)\right\}, \quad \Lambda=\left(\begin{array}{cc}
0 & i \\
-i\left(L+m^{2}\right) & 0
\end{array}\right)
$$

in the energy space $\mathcal{H}=H_{b}^{1} \times L^{2}$, where $H_{b}^{1}$ is the completion of $C_{0}^{\infty}(\Omega)$ in the norm

Then $\Lambda$ with domain

$$
\left\|f_{1}\right\|_{H_{b}^{1}}^{2}=\int\left\{\left|\nabla_{b} f_{1}\right|^{2}+\left(c(x)+m^{2}\right)\left|f_{1}\right|^{2}\right\} d x .
$$

$$
\mathcal{D}(\Lambda)=\left\{f_{1} \in H_{b}^{1} ; \Delta_{b} f_{1} \in L^{2}\right\} \times\left\{f_{2} \in H_{b}^{1} \cap L^{2}\right\}
$$

forms a selfadjoint operator in $\mathcal{H}$, and its resolvent is given by

$$
\mathcal{R}(z)=\left(L+m^{2}-z^{2}\right)^{-1}\left(\begin{array}{cc}
z & i \\
-i\left(L+m^{2}\right) & z
\end{array}\right) .
$$

Let $A: \mathcal{H} \rightarrow \mathcal{H}_{1}=L^{2}$ be defined by

$$
A f=\min \left\{\sqrt{\mu(r)}, r^{-1}\right\} \sqrt{L+m^{2}} f_{1} \quad \text { for } f=\left\{f_{1}, f_{2}\right\} \in \mathcal{H} .
$$

Then the adjoint operator $A^{*}$ is given by

$$
A^{*} g=\left\{{\sqrt{L+m^{2}}}^{-1} \min \left\{\sqrt{\mu(r)}, r^{-1}\right\} g, 0\right\} \quad \text { for } g \in L^{2} .
$$

Proof of Theorem 2(ii) and (iii). By definition

$$
A \mathcal{R}(z) A^{*} g=\min \left\{\sqrt{\mu(r)}, r^{-1}\right\} z\left(L+m^{2}-z^{2}\right)^{-1} \min \left\{\sqrt{\mu(r)}, r^{-1}\right\} g
$$


for $g \in \mathcal{D}\left(A^{*}\right)$. Then since

$$
\begin{aligned}
& \int\left|\min \left\{\sqrt{\mu(r)}, r^{-1}\right\} z\left(L+m^{2}-z^{2}\right)^{-1} f\right|^{2} d x \\
& \leq m^{2} \int r^{-2}\left|\left(L+m^{2}-z^{2}\right)^{-1} f\right|^{2} d x+\int \mu\left|-m^{2}+z^{2}\right|\left|\left(L+m^{2}-z^{2}\right)^{-1} f\right|^{2} d x,
\end{aligned}
$$

using Theorem 1(ii) and (iii), we obtain

$$
\left\|A \mathcal{R}(z) A^{*} g\right\| \leq \sqrt{m^{2} C_{1}+C_{2}}\|g\| .
$$

We now return to Proposition 3. Then (14) shows that

$$
\begin{aligned}
\left|\int_{0}^{ \pm \infty}\left\|A e^{-i t \Lambda} f\right\|^{2} d t\right| & =\left|\int_{0}^{ \pm \infty}\left\|\min \left\{\sqrt{\mu(r)}, r^{-1}\right\} \sqrt{L+m^{2}} w(t)\right\|^{2} d t\right| \\
& \leq 2 \sqrt{m^{2} C_{1}+C_{2}}\|f\|_{\mathcal{H}}^{2} .
\end{aligned}
$$

Since

$$
w(t)=\cos \left(t \sqrt{L+m^{2}}\right) f_{1}+{\sqrt{L+m^{2}}}^{-1} \sin \left(t \sqrt{L+m^{2}}\right) f_{2},
$$

this inequality implies (ii).

To show (iii) we have only to use Theorem $1(\mathrm{i})$ with $b$ and $c$ identically equal to 0 . In fact, let $\Lambda_{0}$ represent the operator $\Lambda$ with $L=L_{0}$ and $m=0$, and let $\mathcal{R}_{0}(z)$ be its resolvent. Then choosing

$$
A_{0} f=\sqrt{\mu(r)} \sqrt{L_{0}} f_{1},
$$

we have corresponding to (15)

$$
A_{0} \mathcal{R}_{0}(z) A_{0}^{*} g=\sqrt{\mu(r)} z\left(L_{0}-z^{2}\right)^{-1} \sqrt{\mu(r)} g
$$

and hence

$$
\left\|A_{0} \mathcal{R}_{0}(z) A_{0}^{*} g\right\| \leq 2 \sqrt{5}\|\mu\|_{L^{1}}\|g\|
$$

Thus, following the above argument leads us to the conclusion.

\section{Acknowledgements}

The author would like to express his gratitude to the referees for carefully reading the manuscript and making valuable suggestions.

\section{References}

[1] M. Ben-Artzi and S. Klainerman, Decay and regularity for Schrödinger equation, J. Anal. Math. 58 (1992), 25-37. Zbl 0802.35057 MR 1226935

[2] S. Cuccagna and P. P. Schirmer, On the wave equation with magnetic potential, Comm. Pure Appl. Math. 54 (2001), 135-152. Zbl 1032.35122 MR 1794351 
[3] P. D'Ancona and L. Fanelli, Strichartz and smoothing estimates for dispersive equations with magnetic potentials, Comm. Partial Differential Equations 33 (2008), 1082-1112. Zbl 1160.35363 MR 2424390

[4] M. B. Erdogan, M. Goldberg and W. Schlag, Strichartz and smoothing estimates for Schrödinger operators with large magnetic potentials in $\mathbb{R}^{3}$, J. Eur. Math. Soc. 10 (2008), 507-531. Zbl 1152.35021 MR 2390334

[5] V. Georgiev, A. Stefanov and M. Tarulli, Smoothing - Strichartz estimates for the Schrödinger equation with small magnetic potential, Discrete Contin. Dynam. Systems $\mathbf{1 7}$ (2007), 771-786. Zbl 1125.35077 MR 2276474

[6] T. Ikebe and J. Uchiyama, On the asymptotic behavior of eigenfunctions of second-order elliptic operators, J. Math. Kyoto Univ. 11 (1971), 425-448. Zbl 0233.35021 MR 0289967

[7] H. Kalf, U.-W. Schmincke, J. Walter and R. Wüst, On the spectral theory of Schrödinger and Dirac operators with strongly singular potentials, in Spectral theory and differential equations (Dundee, 1974), Lecture Notes in Math. 448, Springer, 1975, 182-226. Zbl 0311.47021 MR 0397192

[8] T. Kato, Wave operators and similarity for some non-selfadjoint operators, Math. Ann. 162 (1966), 258-279. Zbl 0139.31203 MR 0190801

[9] T. Kato and K. Yajima, Some examples of smooth operators and the associated smoothing effect, Rev. Math. Phys. 1 (1989), 481-496. Zbl 0833.47005 MR 1061120

[10] S. T. Kuroda, An introduction to scattering theory, Lecture Notes Ser. 51, Aarhus Univ., 1978. Zbl 0407.47003 MR 0528757

[11] K. Mochizuki, Eigenfunction expansions associated with the Schrödinger operator with a complex potential and the scattering inverse problem, Proc. Japan Acad. 43 (1967), 638643. Zbl 0157.21701 MR 0222494

[12] K. Mochizuki, Spectral and scattering theory for second order elliptic differential operators in an exterior domain, Lecture Notes Univ. Utah, Winter and Spring 1972.

[13] K. Mochizuki, Scattering theory for wave equations with dissipative term, Publ. Res. Inst. Math. Sci. 12 (1976), 383-390. Zbl 0357.35067 MR 0437955

[14] K. Mochizuki, Scattering Theory for Wave Equations, Kinokuniya, 1983 (in Japanese).

[15] K. Watanabe, Smooth perturbations of the selfadjoint operator $|\Delta|^{\alpha / 2}$, Tokyo J. Math. 14 (1991), 239-250. Zbl 0757.47015 MR 1108171

[16] K. Yajima, Schrödinger evolution equation with magnetic fields, J. Anal. Math. 56 (1991), 29-76. Zbl 0739.35083 MR 1243098 